\title{
Dinâmica do Surgimento de Brotos de Alfafa em Função de Diferentes Fontes de Fósforo, da Aplicação de Gesso e do Momento de Calagem ${ }^{1}$
}

\author{
Patrícia Sarmento ${ }^{2}$, Moacyr Corsi ${ }^{3}$, Fábio Prudêncio de Campos ${ }^{4}$
}

\begin{abstract}
RESUMO - O fósforo (P) é um importante nutriente para o surgimento de brotos em alfafa. Através do estudo da dinâmica do aparecimento de brotos pode-se obter informações que auxiliam na adoção de um manejo eficiente. Diversas fontes de P estão disponíveis no mercado, sendo que a eficiência deste nutriente é afetada pela acidez do solo. O uso do gesso com fosfato de rocha pode corrigir o perfil do solo em relação ao alumínio e diminuir a fixação de P. Num experimento conduzido em vasos, superfosfato triplo (ST), fosfato de Gafsa (FG) e FG com gesso, aplicados antes e depois da calagem, foram avaliados para se estudar o número de brotos laterais e basais em alfafa, assim como a dinâmica do surgimento destes brotos em função das seguintes doses: 0, 50, 100 e $200 \mathrm{mg} \mathrm{P} \mathrm{dm}^{-3}$. A avaliação envolveu três cortes da cultura. A dose de $100 \mathrm{mg} \mathrm{P} \mathrm{dm}^{-3}$ retardou em 6 a 15 dias o surgimento do segundo broto basal quando comparada à dose de $200 \mathrm{mg} \mathrm{P} \mathrm{dm}^{-3}$. Os brotos laterais surgiram 24 dias após o aparecimento dos brotos basais quando empregou-se a dose de 200 $\mathrm{mg} \mathrm{P} \mathrm{dm}{ }^{-3}$. A adição de fósforo aumentou o número de brotos basais e laterais de 1,5 e 0,5 broto/planta para 8,0 e 6,9 brotos/planta, respectivamente. Verificou-se um maior número de hastes basais com a utilização do FG ( 5,1 brotos/planta) do que com o uso de ST (2,9 brotos/planta). Não houve efeito do gesso sobre os brotos laterais. O uso do FG mais gesso resultou em 5,9 brotos basais/planta, enquanto que o uso do FG não combinado com gesso resultou em 3,9 brotos basais/planta. Não houve efeito do momento de calagem sobre o número de brotos da alfafa.
\end{abstract}

Palavras-chave: broto basal, broto lateral, calagem, fosfato de Gafsa, gesso, Medicago sativa L.

\section{Shooting Emergence Dynamics in Alfalfa as a Function of Different Phosphorus Sources, Use of Gypsum and Liming Time}

\begin{abstract}
Phosphorus (P) is an important nutrient for the emergence of shoots in alfalfa and its efficiency is affected by soil acidity. Shooting emergence dynamics studies provide helpful information for the adoption of an efficient management. Several $P$ sources are available in the market. The use of gypsum with phosphate rock can correct the soil profile for aluminum, as well as decrease the $\mathrm{P}$ fixation by the soil. In an experiment under greenhouse conditions aiming to study the number of shoots, as well as lateral and basal shooting emergence dynamics in alfalfa, Triple superphosphate (TS), Gafsa phosphate (GP) and GP with gypsum, applied before and after liming, were evaluated at the following rates during three harvests: $0,50,100$ and $200 \mathrm{mg} \mathrm{P} \mathrm{dm}^{-3}$. The $100 \mathrm{mg} \mathrm{P} \mathrm{dm}^{-3}$ rate delayed the emergence of the second basal shoot by 6 to 15 days, when compared to the $200 \mathrm{mg} \mathrm{P} \mathrm{dm}^{-3}$. The lateral shoots appeared 24 days after the emergence of the basal ones for the $200 \mathrm{mg} \mathrm{P} \mathrm{dm}^{-3}$ treatment. The increase in phosphorus rates increased the number of basal and lateral shoots from 1.5 and 0.5 shoots/plant to 8.0 and 6.9 shoots/plant, respectively. A greater number of basal shoots was verified when PG was used (5.1 shoots/plant), when compared to the use of TS (2.9 shoots/plant). No effect of gypsum on the lateral shooting was verified. The treatment combining GP and gypsum yielded 5.9 basal shoots/plant while the GP treatment yielded 3.9. There was no effect of liming time on the number of shoots in alfalfa.
\end{abstract}

Key Words: basal shoot, lateral shoot, liming, Gafsa phosphate, gypsum, Medicago sativa L.

\section{Introdução}

O fósforo é encontrado em diversas fontes disponíveis no mercado, que variam entre si em concentração e solubilidade (Van Raij et al., 1982). Os fosfatos solúveis são as melhores formas de adubo fosfatado, sendo também os mais onerosos. No entanto, os fosfatos de rocha reativos, como o fosfato de Gafsa, têm apresentado eficiência semelhante àquela dos fosfatos solúveis, devendo ser, portanto, uma fonte de fósforo mais econômica. $\mathrm{O}$ fosfato de Gafsa necessita da acidez do solo para solubilizar-se e, por conseguinte, precisa ser aplicado antes da calagem, seguido de um período de incubação (Van Raij et al., 1982). O uso do

\footnotetext{
${ }_{1}$ Parte da Dissertação de Mestrado do primeiro autor apresentada à USP/ESALQ. Projeto financiado pela FAPESP

2 Doutoranda do Dept. de Zootecnia - FCAV/UNESP, Via de acesso Prof. Paulo Donato Castellani, s/n km 5 - $14884-900$ - Jaboticabal. E.mail: psarment001@yahoo.com.br

${ }^{3}$ Prof. Titular do Depart. de Produção Animal USP/ESALQ, C.P. 9 - CEP: 13.418-900 - Piracicaba. E.mail: moa@esalq.usp.br

${ }^{4}$ Pós-Doutorando - Depart. de Produção Animal - USP/ESALQ. E.mail: fpcampos_99@yahoo.com
} 
fosfato de Gafsa mais gesso pode ser uma alternativa viável para corrigir o perfil do solo em relação ao alumínio, diminuindo a fixação do fósforo pelo solo e aumentando, conseqüentemente, a eficiência do adubo.

Adicionalmente, o gesso apresenta-se como fonte de enxofre para as plantas, corrigindo as deficiências desse elemento nas forrageiras que recebem fontes de fósforo isentas de enxofre em sua composição (Faquin et al., 1997).

Segundo Sanderson \& Jones (1993), o fósforo é um importante nutriente para o surgimento de brotos de alfafa (Medicago sativa L.). Esses mesmos autores constataram um aumento de 3,8 para 4,7 brotos por planta de alfafa com o aumento da dose de fósforo de 0 para $59 \mathrm{~kg} \mathrm{P} \mathrm{ha}^{-1}$. O número de brotos é considerado um dos componentes da produção de forragem (Fick et al., 1988), sendo utilizado como um indicador de vigor e persistência das pastagens (Da Silva \& Pedreira, 1997).

Segundo Teuber \& Brick (1988), a primeira haste da alfafa a surgir após a germinação é a primária. Os brotos subseqüentes que surgem de gemas na base da planta são as hastes secundárias, formando a coroa primária. Quando as plantas do primeiro crescimento não são cortadas, hastes terciárias, ou laterais, podem surgir eventualmente, a partir das gemas axilares das folhas de hastes primárias e secundárias. Nas rebrotas os brotos originam-se de gemas da coroa, formando as hastes da coroa, sendo que as gemas axilares das hastes remanescentes darão origem aos brotos axilares (Romero et al., 1995). Segundo Gosse et al. (1988), as diferentes categorias de brotos de alfafa diferem quanto às taxas de crescimento e senescência. Leach (1970) observou que 90\% da produção de matéria seca são atribuídos a brotos que surgiam da coroa ou de pontos situados até dois centímetros acima dela.

Cowett \& Sprague afirmavam, desde 1962, que são vários os fatores que afetam o número de brotos por planta de alfafa, como teor de água no solo, intensidade luminosa, temperatura, altura e freqüência de corte, assim com a nutrição mineral. Já a proporção de brotos basais e laterais depende do momento do corte anterior (Juan, citado por Romero et al.,1995), da altura ou freqüência de corte e do cultivar utilizado (Monteiro, 1989; Belesky \& Fedders, 1997).

Para o aperfeiçoamento do sistema de manejo de corte ou pastejo da alfafa, faz-se necessário o entendimento do comportamento fisiológico e morfológico da planta. O estudo da dinâmica populacional dos brotos e o número de brotos da alfafa em diferentes níveis de fertilidade do solo colaboram para a obtenção de informações básicas para a adoção de um manejo eficiente da alfafa.

O objetivo deste trabalho foi avaliar o efeito do superfosfato triplo e do fosfato de Gafsa, associado ou não ao gesso, aplicados em doses crescentes de $P$ antes e após a calagem, na dinâmica de surgimento e no número de brotos basais e laterais de alfafa, durante três períodos de crescimento.

\section{Material e Métodos}

O experimento foi conduzido em estufa na ESALQ (Escola Superior de Agricultura "Luiz de Queiroz"), localizada no município de Piracicaba - SP. O solo utilizado foi coletado no Instituto de Zootecnia de Nova Odessa (SP) e apresentava textura média arenosa. Tal solo foi classificado como LATOSSOLO VERMELHO-AMARELO Alumínico (Typic Mapluolox), apresentando as seguintes características químicas: $\mathrm{pH}=4,1 ; \mathrm{MO}=18,6 \mathrm{mg} \mathrm{dm}^{-3}$; $\mathrm{P}$ (resina) $=3,0 \mathrm{~g} \mathrm{dm}^{-3} ; \mathrm{S}_{-} \mathrm{SO}_{4}=31,9 \mathrm{~g} \mathrm{dm}^{-3}$; $\mathrm{K}=0,5$ mmolc dm ${ }^{-3} ; \mathrm{Ca}=6,0$ mmolc $\mathrm{dm}^{-3} ; \mathrm{Mg}=$ 3,0 mmolc dm ${ }^{-3} ; \mathrm{Al}=9,0$ mmolc dm $\mathrm{dm}^{-3} ; \mathrm{H}+\mathrm{Al}=$ $38,1 \mathrm{mmolc} \mathrm{dm}^{-3} ; \mathrm{T}=47,6 \mathrm{mmolc} \mathrm{dm}^{-3} \mathrm{e} \mathrm{V}=20,0 \%$.

Os tratamentos utilizados foram: superfosfato triplo aplicado antes da calagem (STAC), superfosfato triplo depois da calagem (STDC), fosfato de Gafsa antes da calagem (FGAC), fosfato de Gafsa depois da calagem (FGDC), fosfato de Gafsa antes da calagem mais gesso (FGACG) e fosfato de Gafsa depois da calagem mais gesso (FGDCG). Foram utilizadas as doses de $0,50,100$ e $200 \mathrm{mg} \mathrm{P} \mathrm{dm}^{-3}$. O superfosfato triplo e o fosfato de Gafsa apresentaram 47,11 e $27,39 \%$ de $\mathrm{P}_{2} \mathrm{O}_{5}$ total e 40,02 e $11,06 \%$ de $\mathrm{P}_{2} \mathrm{O}_{5}$ solúvel em ácido cítrico, respectivamente.

As adubações com micronutrientes e $\mathrm{KCl}$ foram realizadas juntamente com a primeira semeadura, usando-se as seguintes concentrações: $171 \mathrm{mg}$ $\mathrm{K} \mathrm{dm}^{-3} ; 1 \mathrm{mg} \mathrm{B} \mathrm{dm}^{-3} ; 0,1 \mathrm{mg} \mathrm{Co} \mathrm{dm}^{-3} ; 1,5 \mathrm{mg} \mathrm{Cu}$ $\mathrm{dm}^{-3} ; 5 \mathrm{mg} \mathrm{Fe} \mathrm{dm}{ }^{-3} ; 5 \mathrm{mg} \mathrm{Mn} \mathrm{dm}{ }^{-3} ; 0,1 \mathrm{mg}$ Mo $\mathrm{dm}^{-3}$ e $5 \mathrm{mg} \mathrm{Zn} \mathrm{dm}^{-3}$. As adubações de manutenção, realizadas após cada corte, consistiram na aplicação de $\mathrm{KCl}$ de acordo com o teor de $\mathrm{K}$ na MS, considerado como sendo 3,6\%. Assim, após a determinação da produção de MS por tratamento, calculou-se a adubação potássica. A calagem, utilizando-se calcário dolomítico, foi realizada 60 dias

R. Bras. Zootec., v.31, n.3, p.1103-1116, 2002 
antes ou após a aplicação dos adubos fosfatados, visando elevar a saturação por bases para $85 \%$. Quando se utilizou gesso (1/3 do Ca adicionado como calcário) juntamente com o FG, a dose de calcário adicionada correspondeu a $2 / 3$ da utilizada no tratamento que não recebeu gesso. Aplicou-se enxofre elementar nos tratamentos que não receberam gesso para manter-se o equilíbrio no fornecimento de enxofre.

A semeadura foi realizada em 12/04/98, cinco meses após a aplicação dos tratamentos. As sementes da alfafa, cultivar XAI 32, foram inoculadas com Sinorhizobium meliloti SEMIA 116, sendo que cinco plantas foram cultivadas em cada vaso. O primeiro corte da alfafa foi realizado quando as plantas tinham 90 dias $(10 / 07 / 98)$ e os dois últimos cortes foram feitos a cada 30 dias (09/08/98 e 08/09/98, respectivamente), cortando-se as plantas a 7,5 cm do solo. A temperatura ambiental média durante o período experimental foi $19^{\circ} \mathrm{C}$, enquanto as temperaturas ambientais máximas e mínimas foram, em média, 26 e $13^{\circ} \mathrm{C}$, respectivamente.

A irrigação dos vasos com água de torneira foi realizada para manter a capacidade de campo do solo em $70 \%$. Os vasos foram pesados diariamente para que a água utilizada pelo sistema solo-planta fosse reposta.

O surgimento dos brotos basais e laterais da alfafa foi avaliado mediante a colocação de fios coloridos a cada dois dias durante os três períodos de crescimento. No presente trabalho, as hastes primárias e as hastes secundárias, originadas da base da planta, assim como os brotos surgidos da coroa durante a rebrota, foram considerados brotos basais. Por outro lado, tanto os brotos surgidos da haste primária e das hastes secundárias, como aqueles originados das hastes remanescentes após o corte, foram considerados brotos laterais.

O delineamento estatístico utilizado foi o de blocos completos ao acaso com parcelas subdivididas no tempo com três repetições. As parcelas foram representadas pelos tratamentos compostos por diferentes fontes de fósforo, com ou sem a adição de gesso, pelo momento de calagem e também pelas doses de $\mathrm{P}$, enquanto que as subparcelas foram representadas pelos cortes.

Os dados foram submetidos à análise de variância utilizando-se o programa SANEST/USP (Sarriés et al., 1993). Para a comparação entre os tratamentos compostos por diferentes fontes de fósforo, associado à adição ou não de gesso e ao momento de calagem, utilizou-se o teste $t$ para contrastes ortogonais. Para a avaliação das doses de fósforo utilizou-se a análise de regressão, enquanto que o teste Tukey foi utilizado para a avaliação dos cortes. As variáveis número de brotos basais e número de brotos laterais foram analisadas aplicando-se a transformação por raiz quadrática. Quanto à dinâmica do surgimento de brotos da alfafa utilizou-se a análise descritiva para discussão dos resultados.

\section{Resultados e Discussão}

Nos tratamentos testemunha ocorreram mortes de plantas, após o primeiro corte, devido à ausência da adubação fosfatada. Em decorrência disso, não se analisou o surgimento de brotos de alfafa nesses tratamentos.

\section{Dinâmica do surgimento de brotos basais}

O segundo broto basal surgiu da primeira folha unifoliolada quando as plantas tinham, em média, 41 dias, para todos tratamentos envolvendo todas as fontes de fósforo na dose de $200 \mathrm{mg} \mathrm{P} \mathrm{dm}^{-3}$ (Figura 1). O segundo broto basal da alfafa no tratamento que recebeu $100 \mathrm{mg} \mathrm{P} \mathrm{dm}^{-3}$ teve seu aparecimento retardado em 6 a 15 dias, quando comparado ao tratamento empregando a dose de $200 \mathrm{mg} \mathrm{P} \mathrm{dm}^{-3}$ (Figura 1). Nos tratamentos que utilizaram fosfato de Gafsa com e sem gesso (Figura 1), o aparecimento dos brotos basais para a dose de $100 \mathrm{mg} \mathrm{P} \mathrm{dm}^{-3}$ iniciou-se no mesmo período em que os brotos surgiram nos tratamentos utilizando $200 \mathrm{mg} \mathrm{P} \mathrm{dm}^{-3}$. Em todos os tratamentos utilizando 100 e $200 \mathrm{mg}$ $\mathrm{P} \mathrm{dm}{ }^{-3}$ verificou-se um aumento no número de brotos basilares até os 90 dias de idade das plantas, quando foi feito o primeiro corte, com exceção do tratamento utilizando superfosfato triplo na dose de $100 \mathrm{mg} \mathrm{P} \mathrm{dm}^{-3}$ aplicado antes da calagem, o qual promoveu um aumento pouco expressivo (Figura 1). Isso provavelmente ocorreu devido à fixação do fósforo proveniente da fonte solúvel adicionada antes da calagem quando comparada àquela adicionada após a calagem. Segundo Van Raij et al. (1982), a aplicação da calagem antes da adubação fosfatada diminui a capacidade de adsorção ou fixação do fósforo pelo solo. O surgimento do segundo broto basal no tratamento com $50 \mathrm{mg} \mathrm{P} \mathrm{dm}^{-3}$ ocorreu, com o uso do fosfato de Gafsa com gesso adicionado após a calagem, quando as plantas tinham em média 50 dias de idade (Figura 1).

Observou-se redução no número de brotos basais

R. Bras. Zootec., v.31, n.3, p.1103-1116, 2002 

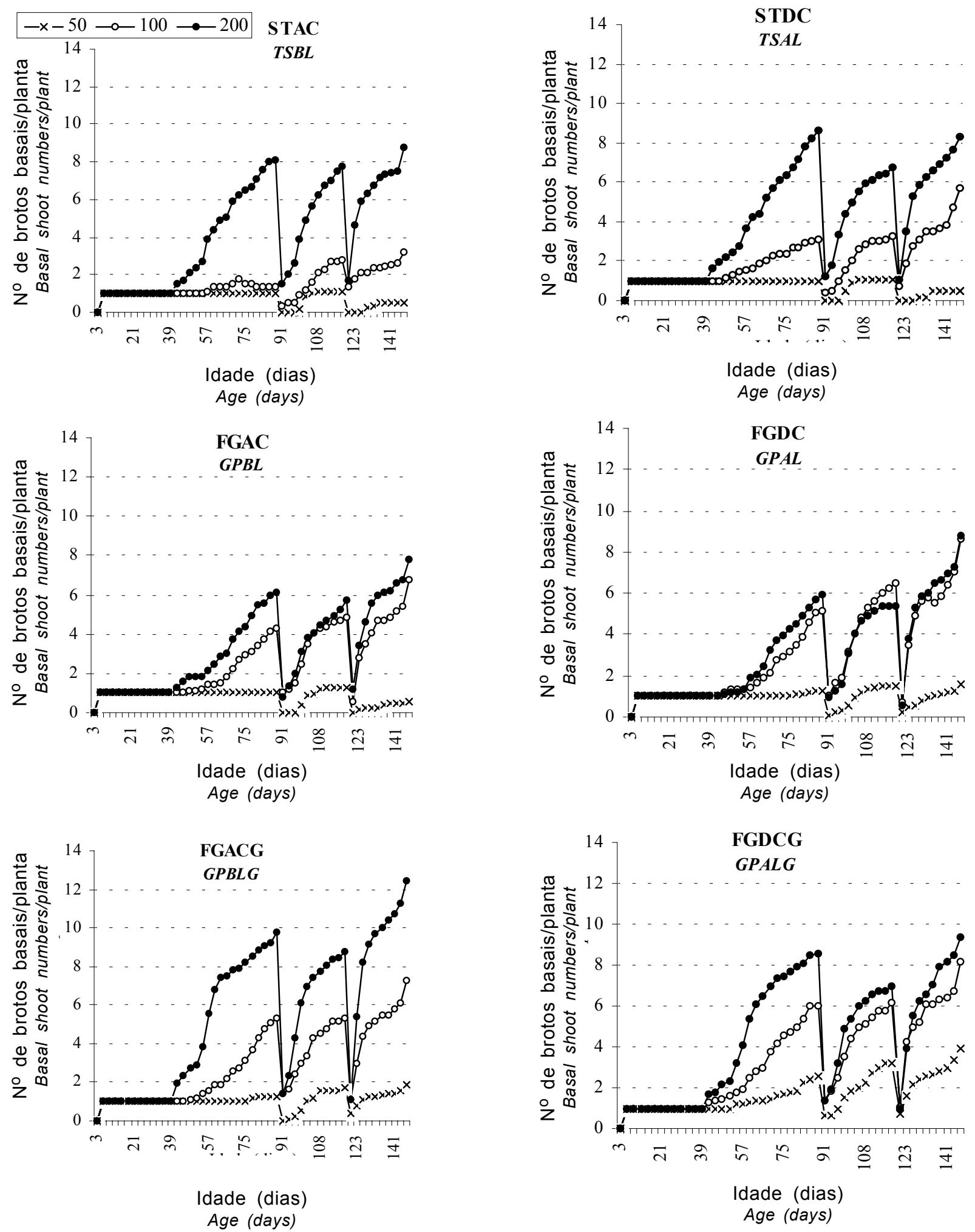

Figura 1 - Dinâmica de surgimento de brotos basais/planta, em função de doses de fósforo em três períodos de crescimento, seguidos de cortes da planta (alfafa).

(STAC e STDC: super triplo antes e depois da calagem; FGAC e FGDC: fosfato de Gafsa antes e depois da calagem; FGACG e FGDCG: fosfato de Gafsa antes e depois da calagem mais gesso).

Figure 1 - Basal shoots emergence dynamic (basal shoots/plants) as a function of phosphorus rates during three growth periods followed by cuts of the plant (alfalfa).

(TSBL, TSAL: triple superphosphate before and after liming; GPBL, GPAL: Gafsa phosphate before and after liming; GPBLG, GPALG: Gafsa phosphate before and after liming combined with gypsum).

R. Bras. Zootec., v.31, n.3, p.1103-1116, 2002 
da alfafa logo em seguida aos cortes (Figura 1). Segundo Monteiro \& Moraes (1996), isso deve-se à alfafa apresentar hábito de crescimento ereto com alongamento do colmo, o que expõe seus pontos de crescimento (meristemas apicais), deixando-os sujeitos à decapitação pelo corte e causando uma redução drástica da área foliar.

Após os cortes, o número de brotos basilares aumentou com a idade das plantas para os tratamentos com doses de 100 e $200 \mathrm{mg} \mathrm{P} \mathrm{dm}^{-3}$ em todas as fontes de fósforo utilizadas (Figura 1). Estes resultados estão de acordo com os encontrados por Juan, citado por Romero et al. (1995), que verificou um aumento dos brotos basais em observações realizadas até 42 dias após a rebrota. As brotações subseqüentes da coroa são conseqüência da quebra de dominância apical provocada pela decapitação do meristema apical durante o corte (Monteiro \& Moraes, 1996).

A rebrota das hastes da coroa foi mais rápida para as doses mais elevadas de fósforo. Entretanto, o tratamento que utilizou o fosfato de Gafsa na dose de $100 \mathrm{mg} \mathrm{P} \mathrm{dm}{ }^{-3}$, associado ou não ao gesso, apresentou resultados próximos aos do tratamento com dose mais alta de fósforo (Figura 1), exceto para o tratamento FGACG.

Em relação ao período de rebrota nos tratamentos com 100 e $200 \mathrm{mg} \mathrm{P} \mathrm{dm}^{-3}$, observou-se que o aparecimento de brotos basais foi mais rápido na segunda que na primeira rebrota (Figura 1). Na segunda rebrota, ocorreu aumento acentuado no número de brotos até, aproximadamente, 9 dias após segundo corte, enquanto na primeira rebrota, até, aproximadamente, 15 dias depois do primeiro corte (Figura 1). Isso pode ter ocorrido em conseqüência do aumento da temperatura ambiental durante o crescimento após o segundo corte, em que as temperaturas máxima, mínima e média semanais, a partir do primeiro corte foram de 22,8 e $15^{\circ} \mathrm{C}$, respectivamente, e na semana a partir da segundo corte, de 28 , $16 \mathrm{e} 22^{\circ} \mathrm{C}$, respectivamente. Este resultado corrobora os obtidos por Leach (1971), que, estudando o efeito da temperatura $\left(15,21,27\right.$ e $\left.33^{\circ} \mathrm{C}\right)$ no crescimento dos brotos de alfafa, observou rebrota inicial mais rápida em plantas crescidas no ambiente com maior temperatura.

Na primeira e, principalmente, na segunda rebrota, próximo ao segundo e terceiro cortes, respectivamente, houve novos aumentos no número de brotos basais da alfafa nas doses de 100 e $200 \mathrm{mg} \mathrm{P} \mathrm{dm}^{-3}$
(Figura 1). Estes resultados estão de acordo com os obtidos por Monteiro (1989). As novas brotações basilares são consideradas sinal de maturidade da forragem e utilizadas como referência para a realização dos cortes da alfafa, devendo ser preservadas para auxiliarem no vigor da rebrota da planta (Monteiro, 1989).

\section{Dinâmica do surgimento de brotos laterais}

No primeiro período de crescimento, o surgimento de broto lateral ocorreu, aproximadamente, aos 65 dias após a semeadura, quando surgiram os primeiros botões florais na alfafa, somente nos tratamentos que receberam $200 \mathrm{mg} \mathrm{P} \mathrm{dm}^{-3}$, independentemente da fonte de fósforo (Figura 2). A exceção foi para o tratamento com fosfato de Gafsa com gesso aplicado após a calagem, na dose de $100 \mathrm{mg} \mathrm{P} \mathrm{dm}^{-3}$, que também apresentou brotação lateral no primeiro período de crescimento (Figura 2), quando surgiram os botões florais no mesmo momento que na dose de $200 \mathrm{mg} \mathrm{P} \mathrm{dm}^{-3}$. Estes brotos laterais aumentaram até o momento do primeiro corte, realizado aos 90 dias de idade.

Nas rebrotas, os brotos laterais originados logo após os cortes surgiram de gemas axilares de hastes remanescentes, enquanto aqueles que apareciam nos últimos 10 dias do ciclo eram originados, em sua maioria, de gemas de folhas trifolioladas de brotos laterais ou dos brotos basais. Esses últimos surgiam com o aparecimento dos botões florais, nas doses de 100 e $200 \mathrm{mg} \mathrm{P} \mathrm{dm}^{-3}$, e apresentavam, aparentemente, menor peso que os brotos basais ou brotos laterais originados de hastes remanescentes.

Na segunda rebrota, o aumento do número de brotos laterais foi mais acentuado que na primeira (Figura 2), à semelhança do que ocorreu para os brotos basais (Figura 1), o que foi devido, provavelmente, ao aumento de temperatura no período do segundo corte e, também, à solubilização do fósforo proveniente do fosfato de Gafsa, com o tempo constatado pelo aumento do teor de fósforo no solo de $15,33 \mathrm{mg} \mathrm{dm}^{-3}$, antes da semeadura para $20,83 \mathrm{mg} \mathrm{dm}^{-3}$, após o último corte. Braga et al. (1991) obtiveram, na dose de $400 \mathrm{~kg} \mathrm{ha}^{-1} \mathrm{de}$ $\mathrm{P}_{2} \mathrm{O}_{5}$, índice de eficiência agronômica do fosfato de Gafsa de 70 e 152 no primeiro e terceiro ciclos de produção de soja (Glycine max L.), respectivamente, em decorrência da solubilização do adubo fosfatado, em LATOSSOLO VERMELHOESCURO textura média. 

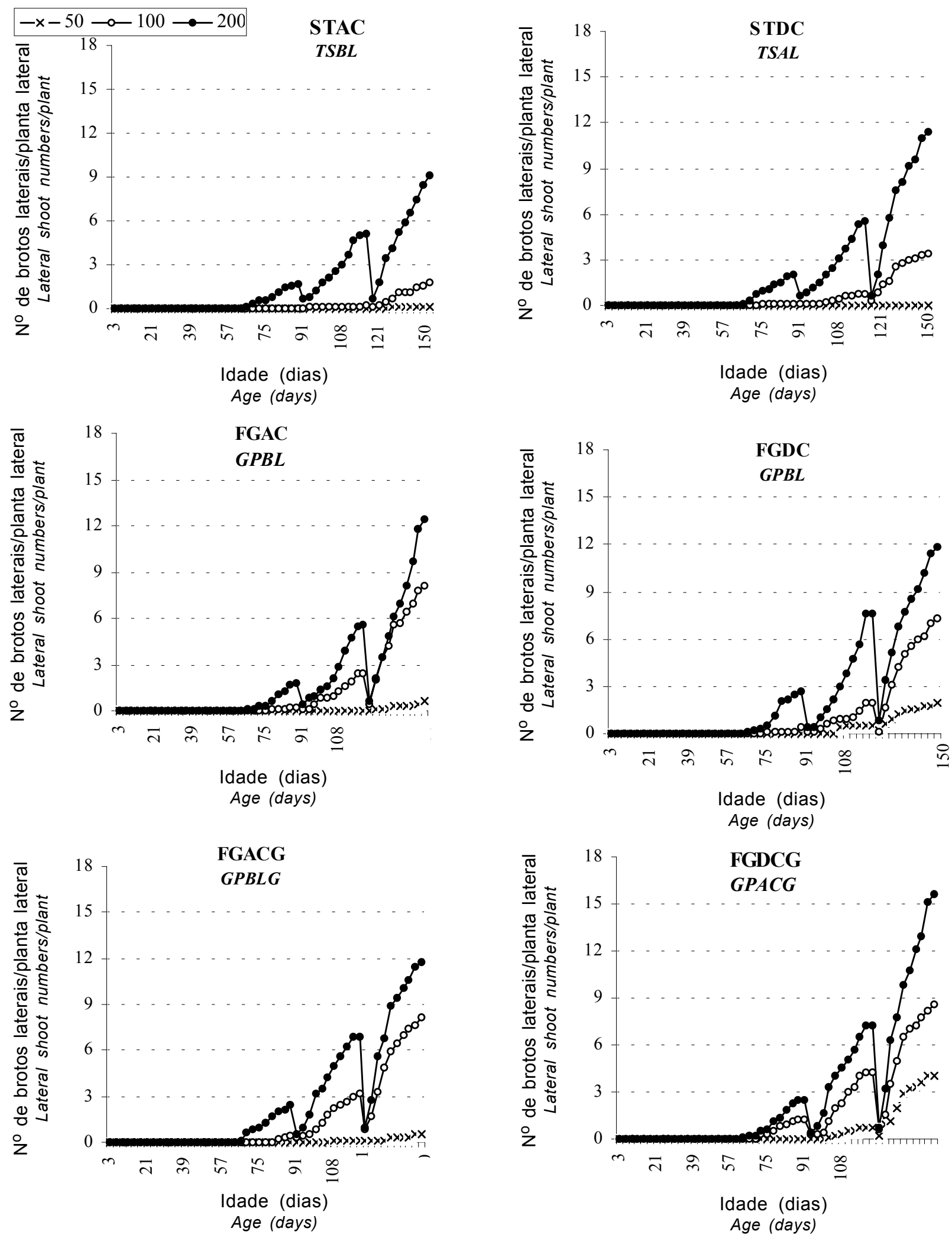

Figura 2 - Dinâmica de surgimento de brotos laterais/planta, em função de doses de fósforo em três períodos de crescimento, seguidos de cortes da planta (alfafa).

(STAC e STDC: super triplo antes e depois da calagem; FGAC e FGDC: fosfato de Gafsa antes e depois da calagem; FGACG e FGDCG: fosfato de Gafsa antes e depois da calagem mais gesso).

Figure 2 - Lateral shoots emergence dynamic (basal shoots/plants) as a function of phosphorus rates during three growth periods followed by cuts of the plant (alfalfa).

(TSBL, TSAL: triple superphosphate before and after liming; GPBL, GPAL: Gafsa phosphate before and after liming; GPBLG, GPALG: Gafsa phosphate before and after liming combined with gypsum).

R. Bras. Zootec., v.31, n.3, p.1103-1116, 2002 
Número de brotos basais e laterais

Foram observadas interações significativas $(\mathrm{P}<0,05)$ entre os tratamentos, fontes e doses de fósforo, cortes e fontes de fósforo, assim como entre cortes e doses de fósforo no número de brotos basais por planta. Para os brotos laterais, foi detectada uma interação tripla $(\mathrm{P}<0,05)$ entre os tratamentos com fontes diferentes de fósforo, doses e cortes.

A adição de fósforo aumentou $(\mathrm{P}<0,05)$ o número de brotos basais e laterais em todas as fontes de fósforo utilizadas nos três períodos de crescimento (Tabela 1 e Figura 3). Estes resultados, semelhantes aos resultados obtidos por Sanderson \& Jones (1993), confirmam o efeito do fósforo como nutriente, o qual estimula o aumento do número de brotos em leguminosas.

$\mathrm{O}$ número de brotos laterais foi maior $(\mathrm{P}<0,05)$ com a utilização do fosfato de Gafsa quando comparado ao ST na dose de $100 \mathrm{mg} \mathrm{P} \mathrm{dm}^{-3}$ para o segundo ciclo de crescimento da planta (Tabela 2 e contraste 6

Tabela 1 - Equações de regressão ajustadas entre o número de brotos basais (médias dos três cortes) e laterais da alfafa $(\mathrm{Y})$ como variável dependente das doses de fósforo $(\mathrm{X})$

Table 1 - Adjusted regression equations relating the basal (means of three cuts) and lateral shoot number of the alfalfa ( $Y$ ) as the dependent variable on the phosphorus rate $(X)$

\begin{tabular}{|c|c|c|}
\hline $\begin{array}{l}\text { Tratamentos com fontes de fósforo } \\
\text { Treatments with phosphorus sources }\end{array}$ & $\begin{array}{l}\text { Equaç̃oes } \\
\text { Equations }\end{array}$ & $\begin{array}{l}\mathrm{R}^{2} \\
R^{2} \\
\end{array}$ \\
\hline $\begin{array}{l}\text { Brotos basais } \\
\text { Basal shoots }\end{array}$ & & \\
\hline $\begin{array}{l}\text { STAC }^{1}(T S B L)^{2} \\
\text { STDC }^{3}(T S A L)^{4} \\
\text { FGAC }^{5}(G P B C)^{6} \\
\text { FGDC }^{7}(G P A L)^{8} \\
\text { FGACG }^{9}(G P B L C)^{10} \\
\text { FGDCG }^{11}(G P A L C)^{12}\end{array}$ & $\begin{array}{l}Y=-1,989+0,0500 X \\
Y=-1,111+0,0460 X \\
Y=0,333+0,0338 X \\
Y=1,478+0,0299 X \\
Y=-0,556+0,0560 X \\
Y=2,489+0,0310 X\end{array}$ & $\begin{array}{l}0,98^{*} \\
0,98^{*} \\
0,77^{*} \\
0,57^{*} \\
0,96^{*} \\
0,84^{*}\end{array}$ \\
\hline $\begin{array}{l}\text { Brotos laterais } \\
\text { Lateral shoots }\end{array}$ & $\begin{array}{l}\text { Corte } 1 \\
\text { Cut } 1\end{array}$ & \\
\hline $\begin{array}{l}\text { STAC (TSBL) } \\
\text { STDC (TSAL) } \\
\text { FGAC }(G P B C) \\
\text { FGDC }(G P A L) \\
\text { FGACG }(G P B L C)\end{array}$ & $\begin{array}{l}\mathrm{Y}=-0,835+0,0119 \mathrm{X} \\
\mathrm{Y}=-0,465+0,0142 \mathrm{X} \\
\mathrm{Y}=-0,800+0,0126 \mathrm{X} \\
\mathrm{Y}=-1,140+0,0186 \mathrm{X} \\
\mathrm{Y}=-1,035+0,017 \mathrm{X}\end{array}$ & $\begin{array}{l}0,89^{*} \\
0,91^{*} \\
0,95^{*} \\
0,96^{*} \\
0,97^{*}\end{array}$ \\
\hline FGDCG $(G P A L C)$ & $\begin{array}{c}\mathrm{Y}=-0,630+0,0163 \mathrm{X} \\
\text { Corte } 2 \\
\text { Cut } 2\end{array}$ & $0,96^{*}$ \\
\hline $\begin{array}{l}\text { STAC (TSBL) } \\
\text { STDC (TSAL) } \\
\text { FGAC (GPBC) } \\
\text { FGDC (GPAL) } \\
\text { FGACG }(G P B L C)\end{array}$ & $\begin{array}{l}\mathrm{Y}=-2,465+0,0364 \mathrm{X} \\
\mathrm{Y}=-2,400+0,0385 \mathrm{X} \\
\mathrm{Y}=-1,565+0,0365 \mathrm{X} \\
\mathrm{Y}=-2,330+0,0489 \mathrm{X} \\
\mathrm{Y}=-1,770+0,0443 \mathrm{X}\end{array}$ & $\begin{array}{l}0,91 * \\
0,96^{*} \\
0,99 * \\
0,98^{*} \\
0,99 *\end{array}$ \\
\hline $\operatorname{FGDCG}(G P A L C)$ & $\begin{array}{c}\mathrm{Y}=-0,770+0,0417 \mathrm{X} \\
\text { Corte } 3 \\
\text { Cut } 3\end{array}$ & $0,94 *$ \\
\hline $\begin{array}{l}\text { STAC (TSBL) } \\
\text { STDC }(T S A L) \\
\text { FGAC }(G P B C) \\
\text { FGDC }(G P A L) \\
\text { FGACG }(G P B L C) \\
\text { FGDCG }(G P A L C) \\
\end{array}$ & $\begin{array}{l}Y=-3,540+0,0616 X \\
Y=-4,000+0,0766 X \\
Y=-1,535+0,0735 X \\
Y=-0,260+0,0627 X \\
Y=-1,270+0,0695 X \\
Y=0,535+0,0764 X\end{array}$ & $\begin{array}{l}0,97^{*} \\
1,00^{*} \\
0,88^{*} \\
0,94^{*} \\
0,85^{*} \\
1,00^{*}\end{array}$ \\
\hline
\end{tabular}

STAC $^{1}$ e STDC 3 : super triplo antes e depois da calagem; FGAC ${ }^{5}$ e FGDC 7 : fosfato de Gafsa antes e depois da calagem; FGACG $^{9}$ e FGDCG ${ }^{11}$ : fosfato de Gafsa antes e depois da calagem mais gesso; ${ }^{*}$ Significativo $(P<0,05)$.

$T S B L^{2}, T S A L^{4}$ : triple superphosphate before and after liming; GPBL ${ }^{6}, G_{A} L^{8}$ : Gafsa phosphate before and after liming; GPBLG ${ }^{10}$, GPALG $^{12}$ : Gafsa phosphate before and after liming combined with gypsum; *Significant $(P<.05)$.

\section{R. Bras. Zootec., v.31, n.3, p.1103-1116, 2002}




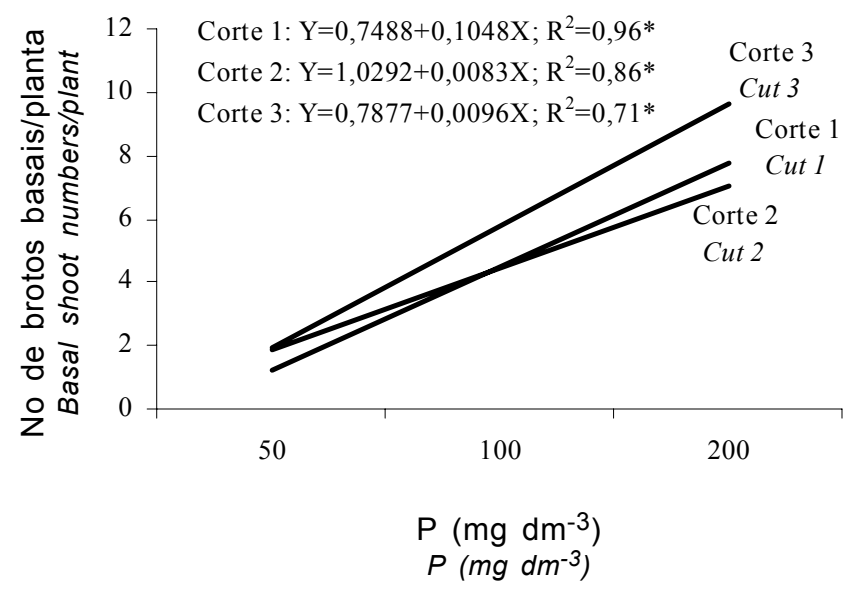

Figura 3 - Número de brotos basais da alfafa, em função das doses de fósforo em três períodos de crescimento (cortes). *significativo $(P<0,05)$.

Figure 3 - Number of basal shoot in alfalfa, as a function of phosphorus rates during three growth periods (cuts). *Significant $(P<.05)$. da Tabela 3) e nas doses de 50 e $100 \mathrm{mg} \mathrm{P} \mathrm{dm}^{-3}$ para o terceiro ciclo de crescimento (Tabela 2 e contraste 11 da Tabela 3). Analisando os tratamentos para as diferentes fontes de fósforo dentro de cada dose, observou-se um maior número de hastes originadas da coroa $(\mathrm{P}<0,05)$ com a utilização do fosfato de Gafsa do que com o uso do superfosfato triplo nas doses de 50 e $100 \mathrm{mg} \mathrm{P} \mathrm{dm}^{-3}$ (Tabela 4 e contraste 1 da Tabela 5). Este fato também foi observado pelo desdobramento da interação entre os tratamentos envolvendo fontes de fósforo e cortes para todos períodos de crescimento (Tabela 4 e contraste 6 da Tabela 5). Estes resultados devem-se, provavelmente, à fixação do fósforo proveniente da fonte solúvel causada pelo atraso da semeadura e à solubilização do fosfato de Gafsa com o passar do tempo.

Não houve efeito $(\mathrm{P}>0,05)$ da utilização de gesso associado ao fosfato de Gafsa sobre o número de brotos laterais da alfafa (Tabela 2 e contrastes $3,8 \mathrm{e}$ 13 da Tabela 3). No entanto, a adição de gesso ao

Tabela 2 - Número de brotos laterais de alfafa obtidos em todos os tratamentos com fontes de fósforo nos três períodos de crescimento (cortes)

Table 2 - Number of lateral shoots in alfalfa obtained in all the treatments with phosphorus source during three growth period (cuts)

\begin{tabular}{|c|c|c|c|c|c|c|}
\hline \multirow[b]{2}{*}{$\begin{array}{l}\text { Doses de fósforo } \\
\text { Phosphorus rates }\end{array}$} & \multicolumn{6}{|c|}{$\begin{array}{l}\text { Tratamentos com fontes de fósforo } \\
\text { Treatments with phosphorus sources }\end{array}$} \\
\hline & $\begin{array}{c}\text { STAC } \\
T S B L\end{array}$ & $\begin{array}{l}\text { STDC } \\
\text { TSAL }\end{array}$ & $\begin{array}{c}\text { FGAC } \\
G P B L\end{array}$ & $\begin{array}{l}\text { FGDC } \\
G P A L\end{array}$ & $\begin{array}{l}\text { FGACG } \\
G P B L G\end{array}$ & $\begin{array}{l}\text { FGDCG } \\
\text { GPALG }\end{array}$ \\
\hline $\begin{array}{l}\left(\mathrm{mg} \mathrm{dm}^{-3}\right) \\
\left(m g \mathrm{dm}^{-3}\right)\end{array}$ & \multicolumn{6}{|c|}{ Brotos laterais/planta } \\
\hline \multicolumn{7}{|l|}{$\begin{array}{l}\text { Corte } 1 \\
\text { Cut } 1\end{array}$} \\
\hline $\begin{array}{l}50 \\
100 \\
200\end{array}$ & $\begin{array}{l}0,00 \\
0,00 \\
1,67\end{array}$ & $\begin{array}{l}0,00 \\
0,07 \\
2,00\end{array}$ & $\begin{array}{l}0,00 \\
0,20 \\
1,80\end{array}$ & $\begin{array}{l}0,00 \\
0,40 \\
2,68\end{array}$ & $\begin{array}{l}0,00 \\
0,40 \\
2,47\end{array}$ & $\begin{array}{l}0,00 \\
1,27 \\
2,53\end{array}$ \\
\hline \multicolumn{7}{|l|}{$\begin{array}{l}\text { Corte } 2 \\
\text { Cut } 2\end{array}$} \\
\hline $\begin{array}{l}50 \\
100 \\
200\end{array}$ & $\begin{array}{l}0,00 \\
0,20 \\
5,13\end{array}$ & $\begin{array}{l}0,00 \\
0,73 \\
5,53\end{array}$ & $\begin{array}{l}0,00 \\
2,47 \\
5,60\end{array}$ & $\begin{array}{l}0,53 \\
1,93 \\
7,65\end{array}$ & $\begin{array}{l}0,13 \\
3,13 \\
6,93\end{array}$ & $\begin{array}{l}0,73 \\
4,27 \\
7,27\end{array}$ \\
\hline \multicolumn{7}{|l|}{$\begin{array}{l}\text { Corte } 3 \\
\text { Cut } 3\end{array}$} \\
\hline $\begin{array}{l}50 \\
100 \\
200\end{array}$ & $\begin{array}{l}0,13 \\
1,73 \\
9,07\end{array}$ & $\begin{array}{c}0,00 \\
3,40 \\
11,40\end{array}$ & $\begin{array}{l}0,60 \\
8,13\end{array}$ & $\begin{array}{l}2,00 \\
7,33\end{array}$ & $\begin{array}{l}0,53 \\
8,20\end{array}$ & $\begin{array}{l}4,07 \\
8,60\end{array}$ \\
\hline & 12,40 & 11,85 & 11,80 & 15,67 & & \\
\hline
\end{tabular}

STAC e STDC: super triplo antes e depois da calagem; FGAC e FGDC: fosfato de Gafsa antes e depois da calagem; FGACG e FGDCG: fosfato de Gafsa antes e depois da calagem mais gesso. CV (parcelas e subparcelas) $=11,42$ e $8,97 \%$, respectivamente. TSBL, TSAL: triple superphosphate before and after liming; GPBL, GPAL: Gafsa phosphate before and after liming; GPBLG, GPALG: Gafsa phosphate before and after liming combined with gypsum. CV (parts and subparts) $=11,42$ and 8,97\%, respectively. 
Tabela 3 - Contrastes ortogonais e valores dos contrastes para comparação dos números de brotos laterais em alfafa nos três períodos de crescimento (cortes)

Table 3 - Orthogonal contrasts and contrast value for the comparison of the numbers of lateral shoots in alfalfa during the three growth period (cuts)

\begin{tabular}{|c|c|c|c|c|}
\hline & \multirow[t]{2}{*}{$\begin{array}{l}\text { Contrastes } \\
\text { Contrasts }\end{array}$} & \multicolumn{3}{|c|}{$\begin{array}{c}\text { Doses de fósforo }\left(\mathrm{mg} \mathrm{dm}^{-3}\right) \\
\text { Phosphorus rates }\left(\mathrm{mg} \mathrm{dm}^{-3}\right)\end{array}$} \\
\hline & & 50 & 100 & 200 \\
\hline $\mathrm{N}^{\mathrm{O}}$ & & \multicolumn{3}{|c|}{$\begin{array}{l}\text { Valor do contraste } \\
\text { Contrasts value }\end{array}$} \\
\hline \multicolumn{5}{|c|}{ Corte 1} \\
\hline \multicolumn{5}{|c|}{ Cut 1} \\
\hline 1 & $\begin{array}{c}2 \mathrm{STAC}+2 \mathrm{STDC}-\mathrm{FGAC}-\mathrm{FGDC}-\mathrm{FGACG}-\mathrm{FGDCG} \\
2 T S B L+2 T S A L-G P B L-G P A L-G P B L C-G P A L C\end{array}$ & 0,00 & $-2,10$ & $-1,39$ \\
\hline 2 & $\begin{array}{c}\mathrm{STAC}-\mathrm{STDC} \\
T S B L-T S A L\end{array}$ & 0,00 & $-0,15$ & $-0,28$ \\
\hline 3 & $\begin{array}{c}\mathrm{FGAC}+\mathrm{FGDC}-\mathrm{FGACG}-\mathrm{FGDCG} \\
G P B L+G P A L-G P B L C-G P A L C\end{array}$ & 0,00 & $-0,41$ & $-0,14$ \\
\hline 4 & $\begin{array}{c}\text { FGAC }-\mathrm{FGDC} \\
G P B L-G P A L\end{array}$ & 0,00 & $-0,27$ & $-0,31$ \\
\hline 5 & $\begin{array}{l}\text { FGACG-FGDCG } \\
G P B L C-G P A L C\end{array}$ & 0,00 & $-0,44$ & $-0,01$ \\
\hline \multicolumn{5}{|c|}{ Corte 2} \\
\hline 6 & $\begin{array}{c}2 \mathrm{STAC}+2 \mathrm{STDC}-\mathrm{FGAC}-\mathrm{FGDC}-\mathrm{FGACG}-\mathrm{FGDCG} \\
2 T S B L+2 T S A L-G P B L-G P A L-G P B L C-G P A L C\end{array}$ & $-1,60$ & $-4,79 *$ & $-1,49$ \\
\hline 7 & $\begin{array}{c}\mathrm{STAC}-\mathrm{STDC} \\
T S B L-T S A L\end{array}$ & 0,00 & $-0,44$ & $-0,09$ \\
\hline 8 & $\begin{array}{c}\mathrm{FGAC}+\mathrm{FGDC}-\mathrm{FGACG}-\mathrm{FGDCG} \\
G P B L+G P A L-G P B L C-G P A L C\end{array}$ & $-0,19$ & $-0,90$ & $-0,18$ \\
\hline 9 & $\begin{array}{c}\text { FGAC }-\mathrm{FGDC} \\
G P B L-G P A L\end{array}$ & $-0,71$ & 0,15 & $-0,42$ \\
\hline 10 & $\begin{array}{c}\text { FGACG-FGDCG } \\
G P B L C-G P A L C\end{array}$ & $-0,47$ & $-0,29$ & $-0,09$ \\
\hline \multicolumn{4}{|c|}{ Corte 3} & \\
\hline 11 & $\begin{array}{c}2 \mathrm{STAC}+2 \mathrm{STDC}-\mathrm{FGAC}-\mathrm{FGDC}-\mathrm{FGACG}-\mathrm{FGDCG} \\
2 T S B L+2 T S A L-G P B L-G P A L-G P B L C-G P A L C\end{array}$ & $-4,22 *$ & $-5,11^{*}$ & $-1,71$ \\
\hline 12 & $\begin{array}{l}\text { STAC }-\mathrm{STDC} \\
T S B L-T S A L\end{array}$ & 0,21 & $-0,58$ & $-0,40$ \\
\hline 13 & $\begin{array}{c}\mathrm{FGAC}+\mathrm{FGDC}-\mathrm{FGACG}-\mathrm{FGDCG} \\
G P B L+G P A L-G P B L C-G P A L C\end{array}$ & $-0,78$ & $-0,28$ & $-0,45$ \\
\hline 14 & $\begin{array}{c}\text { FGAC }-\mathrm{FGDC} \\
G P B L-G P A L\end{array}$ & $-0,72$ & 0,16 & 0,08 \\
\hline 15 & $\begin{array}{l}\text { FGACG-FGDCG } \\
G P B L C-G P A L C\end{array}$ & $-1,25^{*}$ & $-0,08$ & $-0,54$ \\
\hline
\end{tabular}

STAC e STDC: super triplo antes e depois da calagem; FGAC e FGDC: fosfato de Gafsa antes e depois da calageM; FGACG e FGDCG: fosfato de Gafsa antes e depois da calagem mais gesso. *Significativo $(P<0,05)$.

TSBL, TSAL: triple superphosphate before and after liming; GPBL, GPAL: Gafsa phosphate before and after liming; GPBLG, GPALG: Gafsa phosphate before and after liming combined with gypsum. *Significant $(P<0,05)$.

fosfato de Gafsa aumentou o número de brotos basais $(\mathrm{P}<0,05)$ em relação à utilização do fosfato de Gafsa nas doses de 50 e $200 \mathrm{mg} \mathrm{P} \mathrm{dm}{ }^{-3}$ sem a adição de gesso (Tabela 4 e contraste 3 da Tabela 5), o que foi observado para todos os cortes $(\mathrm{P}<0,05)$ (Tabela $4 \mathrm{e}$ contraste 8 da Tabela 5). Isso possivelmente foi uma decorrência do menor valor do $\mathrm{pH}$ do solo quando o gesso não foi utilizado $(5,78)$, em comparação a sua utilização $(6,06)$, e não uma decorrência dos níveis de fósforo disponíveis no solo, uma vez que estes foram semelhantes quando utilizou-se o fosfato de Gafsa com ou sem gesso $\left(15,33 \mathrm{mg} \mathrm{P} \mathrm{dm}{ }^{-3}\right)$. 
Tabela 4 - Número de brotos basais de alfafa obtidos em todos tratamentos com fontes de fósforo em função das doses de fósforo (médias dos cortes) e dos três períodos de crescimento (cortes) (médias das doses de fósforo)

Table 4 - Number of basal shoots in alfalfa obtained in all the treatments with phosphorus sources as a function of phosphorus rates (means of cuts) and of the three growth period (cuts) (means by phosphorus rates)

\begin{tabular}{|c|c|c|c|c|c|c|}
\hline \multirow{3}{*}{$\begin{array}{l}\text { Doses de fósforo } \\
\text { Phosphorus rates }\end{array}$} & \multicolumn{6}{|c|}{$\begin{array}{l}\text { Tratamentos com fontes de fósforo } \\
\text { Treatments with phosphorus sources }\end{array}$} \\
\hline & STAC & STDC & FGAC & FGDC & FGACG & FGDCG \\
\hline & $T S B L$ & $T S A L$ & $G P B L$ & $G P A L$ & $G P B L G$ & $G P A L G$ \\
\hline $\begin{array}{l}\left(\mathrm{mg} \mathrm{dm}^{-3}\right) \\
\left(m g d m^{-3}\right)\end{array}$ & ----- & ---- & \multicolumn{2}{|c|}{ Brotos laterais/planta } & ---- & ----- \\
\hline $\begin{array}{l}50 \\
100 \\
200\end{array}$ & $\begin{array}{c}0,87 \\
2,43 \\
8,2\end{array}$ & $\begin{array}{c}0,87 \\
4,03 \\
7,9\end{array}$ & $\begin{array}{l}0,97 \\
5,33 \\
6,53\end{array}$ & $\begin{array}{l}1,47 \\
6,73 \\
6,83\end{array}$ & $\begin{array}{c}1,63 \\
5,97 \\
10,33\end{array}$ & $\begin{array}{l}3,23 \\
6,77 \\
8,27\end{array}$ \\
\hline \multicolumn{7}{|l|}{ Cortes } \\
\hline $\begin{array}{l}\text { Corte } 1 \\
\text { Cut } 1\end{array}$ & 3,47 & 4,27 & 3,83 & 4,13 & 5,47 & 5,73 \\
\hline $\begin{array}{l}\text { Corte } 2 \\
\text { Cut } 2\end{array}$ & 3,9 & 3,70 & 3,97 & 4,57 & 5,23 & 5,40 \\
\hline $\begin{array}{l}\text { Corte } 3 \\
\text { Cut } 3\end{array}$ & 4,13 & 4,83 & 5,03 & 6,33 & 7,23 & 7,13 \\
\hline
\end{tabular}

STAC e STDC: super triplo antes e depois da calagem; FGAC e FGDC: fosfato de Gafsa antes e depois da calagem; FGACG e FGDCG: fosfato de Gafsa antes e depois da calagem mais gesso. CV (parcelas e subparcelas) = 11,4 e 9,0\%, respectivamente.

TSBL, TSAL: triple superphosphate before and after liming; GPBL, GPAL: Gafsa phosphate before and after liming; GPBLG, GPALG: Gafsa phosphate before and after liming combined with gypsum. CV (plots and subplots) $=11.4$ and $9.0 \%$, respectively.

Rando (1992) verificou um aumento no número de brotos totais por planta de alfafa de 11,3 para 20,8 quando o $\mathrm{pH}\left(\mathrm{H}_{2} \mathrm{O}\right)$ do solo passou de 5,8 para 6,8 . Por outro lado, Delistoianov et al. (1992), comparando o fosfato de Alvorada com o superfosfato triplo, obtiveram melhores resultados de produção de matéria seca de capim pangola (Digitaria decumbens Stent.) em consorciação com sirato (Macroptilium atropurpureum DC. cv. Siratro), soja-perene (Neonotonia wightii Lackey) e centrosema (Centrosema pubescens Benth.) quando o gesso foi combinado ao fosfato natural em LATOSSOLO VERMELHO-ESCURO fase arenosa.

Da mesma forma, não se observou diferença $(\mathrm{P}>0,05)$ na aplicação do fosfato de Gafsa, com ou sem gesso, antes ou após a calagem na avaliação dos brotos laterais e basais (Tabela 2 e contrastes 4, 5, 9, 10, 14 e 15 da Tabela 3; Tabela 4 e contrastes 4, 5, 9 e 10 da Tabela 5). As únicas exceções ocorreram para as doses de $50 \mathrm{mg} \mathrm{P} \mathrm{dm}^{-3}$ do terceiro corte, para os brotos laterais (Tabela 2 e contraste 15 da Tabela 3 ) e basais (Tabela 4 e contraste 5 da Tabela 5), quando o fosfato de Gafsa foi utilizado com o gesso. Isso pode estar relacionado à diminuição da fixação do fósforo pelo alumínio presente no solo. No entanto, este fato contraria as expectativas iniciais pois, segundo Cantarutti et al. (1981), a calagem aplicada após a fosfatagem é a melhor opção, uma vez que propicia o acréscimo de cálcio e magnésio no solo e gera melhores condições de solubilização do fosfato de rocha. Além disso, a acidez é interrompida para que ocorra a eliminação da toxidez de manganês e de alumínio.

Não houve diferença significativa $(\mathrm{P}>0,05)$ entre a aplicação do superfosfato triplo antes ou depois da calagem no número de brotos laterais e basais da alfafa nos três períodos de crescimento estudados (Tabela 2 e contrastes 2, 7 e 12 da Tabela 3; Tabela 4 e contrastes 2 e 7 da Tabela 5), exceto para os brotos basais na dose de $100 \mathrm{mg} \mathrm{P} \mathrm{dm}{ }^{-3}$.

Analisando os ciclos de crescimento da alfafa, observou-se que, em geral, o número de brotos laterais no primeiro período de crescimento foi menor $(\mathrm{P}<0,05)$ que nos períodos posteriores (Tabela 6), o que deve-se ao fato das hastes laterais se originarem unicamente das folhas trifolioladas, no primeiro ciclo de crescimento, e não das hastes remanescentes, confirmando o que foi relatado por Teuber \& Brick (1988). O mesmo não foi observado para as doses de $50 \mathrm{mg} \mathrm{P} \mathrm{dm}{ }^{-3}$ nos tratamentos em que utilizou o 
Tabela 5 - Contrastes ortogonais e valores dos contrastes para comparação dos números de brotos basais em alfafa em função de doses de fósforo (médias dos cortes) e dos três períodos de crescimento (cortes) (médias de doses de fósforo)

Table 5 - Orthogonal contrasts and contrast value for the comparison of the numbers of basal shoot in alfalfa as a function of phosphorus rates (means of cuts) and of the three growth period (cuts) (means by phosphorus rates)

\begin{tabular}{|c|c|c|c|c|}
\hline & \multirow[t]{2}{*}{$\begin{array}{l}\text { Contrastes } \\
\text { Contrasts }\end{array}$} & \multicolumn{3}{|c|}{$\begin{array}{l}\text { Doses de fósforo }\left(\mathrm{mg} \mathrm{dm}^{-3}\right) \\
\text { Phosphorus rates }\left(\mathrm{mg} \mathrm{dm}^{-3}\right)\end{array}$} \\
\hline & & 50 & 100 & 200 \\
\hline $\mathrm{N}^{\mathrm{O}}$ & & \multicolumn{3}{|c|}{$\begin{array}{l}\text { Valor do contraste } \\
\text { Contrasts value }\end{array}$} \\
\hline \multicolumn{5}{|c|}{ Corte 1} \\
\hline \multicolumn{5}{|c|}{ Cut 1} \\
\hline 1 & $\begin{array}{c}2 \mathrm{STAC}+2 \mathrm{STDC}-\mathrm{FGAC}-\mathrm{FGDC}-\mathrm{FGACG}-\mathrm{FGDCG} \\
2 T S B L+2 T S A L-G P B L-G P A L-G P B L C-G P A L C\end{array}$ & $-1,50^{*}$ & $-2,93^{*}$ & 0,13 \\
\hline 2 & $\begin{array}{c}\mathrm{STAC}-\mathrm{STDC} \\
T S B L-T S A L\end{array}$ & 0,03 & $-0,43^{*}$ & 0,05 \\
\hline 3 & $\begin{array}{c}\mathrm{FGAC}+\mathrm{FGDC}-\mathrm{FGACG}-\mathrm{FGDCG} \\
G P B L+G P A L-G P B L C-G P A L C\end{array}$ & $-0,94^{*}$ & $-0,15$ & $-0,96^{*}$ \\
\hline 4 & $\begin{array}{l}\text { FGAC }- \text { FGDC } \\
G P B L-G P A L\end{array}$ & $-0,27$ & $-0,29$ & $-0,03$ \\
\hline \multirow[t]{4}{*}{5} & $\begin{array}{r}\text { FGACG-FGDCG } \\
G P B L C-G P A L C \\
\end{array}$ & $-0,52^{*}$ & $-0,16$ & 0,32 \\
\hline & & & $\begin{array}{c}\text { Cortes } \\
\text { Cuts }\end{array}$ & \\
\hline & & 1 & 2 & 3 \\
\hline & & \multicolumn{3}{|c|}{$\begin{array}{c}\text { Valor do contraste } \\
\text { Contrasts value } \\
\end{array}$} \\
\hline 6 & $\begin{array}{c}2 \mathrm{STAC}+2 \mathrm{STDC}-\mathrm{FGAC}-\mathrm{FGDC}-\mathrm{FGACG}-\mathrm{FGDCG} \\
2 T S B L+2 T S A L-G P B L-G P A L-G P B L C-G P A L C\end{array}$ & $-1,19^{*}$ & $-1,13^{*}$ & $-1,98^{*}$ \\
\hline 7 & $\begin{array}{c}\text { STAC }-\mathrm{STDC} \\
\text { TSBL }- \text { TSAL }\end{array}$ & $-0,24$ & 0,04 & $-0,15$ \\
\hline 8 & $\begin{array}{c}\mathrm{FGAC}+\mathrm{FGDC}-\mathrm{FGACG}-\mathrm{FGDCG} \\
G P B L+G P A L-G P B L C-G P A L C\end{array}$ & $-0,72 *$ & $-0,55^{*}$ & $-0,78^{*}$ \\
\hline 9 & $\begin{array}{c}\text { FGAC-FGDC } \\
G P B L-G P A L\end{array}$ & $-0,09$ & $-0,13$ & $-0,37$ \\
\hline 10 & $\begin{array}{l}\text { FGACG-FGDCG } \\
G P B L C-G P A L C\end{array}$ & $-0,14$ & $-0,10$ & $-0,12$ \\
\hline
\end{tabular}

STAC e STDC: super triplo antes e depois da calagem; FGAC e FGDC: fosfato de Gafsa antes e depois da calageM; FGACG e FGDCG: fosfato de Gafsa antes e depois da calagem mais gesso. * Significativo $(P<0,05)$.

TSBL, TSAL: Triple superphosphate before and after liming; GPBL, GPAL: Gafsa phosphate before and after liming; GPBLG, GPALG: Gafsa phosphate before and after liming combined with gypsum. * Significant $(P<.05)$.

superfosfato triplo adicionado antes e após a calagem (Tabela 6).

Isso possivelmente ocorreu devido à menor quantidade de fósforo aplicada ao solo e à maior fixação do fósforo proveniente da fonte solúvel misturada ao solo, quando comparada ao fosfato natural, resultando em menor disponibilidade de fósforo para a planta desenvolver hastes basais capazes de dar origem a hastes laterais após o corte. Segundo Braga et al. (1991), em solos muito carentes de fósforo há necessidade de aplicações elevadas deste elemento para corrigir as deficiências.
Analisando-se os cortes dentro de cada tratamento de fonte de fósforo, observou-se um aumento significativo $(\mathrm{P}<0,05)$ no número de brotos basais no terceiro corte para os tratamentos compostos por fosfato de Gafsa aplicado após a calagem e fosfato de Gafsa com gesso adicionado antes e após a calagem (Tabela 7). Os demais tratamentos não ocasionaram alterações no número de brotos basais.

Os resultados observados (Tabelas 6 e 7), relacionados ao aumento do número de brotos basais e laterais da alfafa ocorreu, provavelmente, devido à elevação da temperatura e ao incremento da solubi- 
Tabela 6 - Número de brotos laterais da alfafa obtidos nos tratamentos com fontes de fósforo nos três períodos de crescimento (cortes)

Table 6 - Number of lateral shoots in alfalfa obtained in the treatments with phosphorus source during three growth period (cuts)

\begin{tabular}{|c|c|c|c|}
\hline \multirow[b]{2}{*}{$\begin{array}{l}\text { Doses de fósforo }\left(\mathrm{mg} \mathrm{dm}^{-3}\right) \\
\text { Phosphorus rates }\left(\mathrm{mg} \mathrm{dm}^{-3}\right)\end{array}$} & \multicolumn{3}{|c|}{$\begin{array}{c}\text { Cortes } \\
\text { Cuts }\end{array}$} \\
\hline & 1 & 2 & 3 \\
\hline \multicolumn{4}{|l|}{ Super triplo antes da calagem } \\
\hline \multicolumn{4}{|c|}{ Triple superphosphate before liming } \\
\hline 50 & $0,00^{\mathrm{a}}$ & $0,00^{\mathrm{a}}$ & $0,13^{\mathrm{a}}$ \\
\hline 100 & $0,00^{\mathrm{b}}$ & $0,20^{\mathrm{b}}$ & $1,73^{\mathrm{a}}$ \\
\hline 200 & $1,67^{\mathrm{c}}$ & $5,13^{\mathrm{b}}$ & $9,07^{\mathrm{a}}$ \\
\hline \multicolumn{4}{|l|}{ Super triplo depois da calagem } \\
\hline \multicolumn{4}{|l|}{ Triple superphosphate after liming } \\
\hline 50 & $0,00^{\mathrm{a}}$ & $0,00^{\mathrm{a}}$ & $0,00^{\mathrm{a}}$ \\
\hline 100 & $0,07^{\mathrm{b}}$ & $0,73^{\mathrm{b}}$ & $3,40^{\mathrm{a}}$ \\
\hline 200 & $2,00^{\mathrm{c}}$ & $5,53^{\mathrm{b}}$ & $11,40^{\mathrm{a}}$ \\
\hline \multicolumn{4}{|c|}{ Fosfosfato de Gafsa antes da calagem } \\
\hline \multicolumn{4}{|c|}{ Gafsa phosphate before liming } \\
\hline 50 & $0,00^{\mathrm{b}}$ & $0,00^{\mathrm{b}}$ & $0,60^{\mathrm{a}}$ \\
\hline 100 & $0,20^{\mathrm{c}}$ & $2,47^{\mathrm{b}}$ & $8,13^{\mathrm{a}}$ \\
\hline 200 & $1,80^{\mathrm{c}}$ & $5,60^{\mathrm{b}}$ & $12,40^{\mathrm{a}}$ \\
\hline \multicolumn{4}{|c|}{ Fosfosfato de Gafsa depois da calagem } \\
\hline \multicolumn{4}{|c|}{ Gafsa phosphate after liming } \\
\hline 50 & $0,00^{\mathrm{c}}$ & $0,53^{\mathrm{b}}$ & $2,00^{\mathrm{a}}$ \\
\hline 100 & $0,40^{\mathrm{c}}$ & $1,93^{\mathrm{b}}$ & $7,33^{\mathrm{a}}$ \\
\hline 200 & $2,68^{\mathrm{c}}$ & $7,65^{\mathrm{b}}$ & $11,85^{\mathrm{a}}$ \\
\hline \multicolumn{4}{|c|}{ Fosfosfato de Gafsa antes da calagem + gesso } \\
\hline \multicolumn{4}{|c|}{ Gafsa phosphate before liming with gypsum } \\
\hline 50 & $0,00^{\mathrm{b}}$ & $0,13^{\mathrm{ab}}$ & $0,53^{\mathrm{a}}$ \\
\hline 100 & $0,40^{\mathrm{c}}$ & $3,13^{\mathrm{b}}$ & $8,20^{\mathrm{a}}$ \\
\hline 200 & $2,47^{\mathrm{c}}$ & $6,93^{\mathrm{b}}$ & $11,80^{\mathrm{a}}$ \\
\hline \multicolumn{4}{|c|}{ Fosfosfato de Gafsa depois da calagem + gesso } \\
\hline \multicolumn{4}{|c|}{ Gafsa phosphate after liming with gypsum } \\
\hline 50 & $0,00^{\mathrm{c}}$ & $0,73^{\mathrm{b}}$ & $4,07^{\mathrm{a}}$ \\
\hline 100 & $1,27^{\mathrm{c}}$ & $4,27^{\mathrm{b}}$ & $8,60^{\mathrm{a}}$ \\
\hline 200 & $2,53^{\mathrm{c}}$ & $7,27^{\mathrm{b}}$ & $15,67^{\mathrm{a}}$ \\
\hline
\end{tabular}

* Médias seguidas por letras distintas na mesma linha diferem entre si a $5 \%$ de probabilidade pelo teste de Tukey. CV (parcelas e subparcelas) $=24,6$ e 20,9\%, respectivamente.

${ }^{*}$ Means followed by different letters within lines differ by Tukey test $(P<.05)$. CV (plots and subplots) $=24.6$ and $20.9 \%$, respectively.

lidade do fosfato de Gafsa com o passar do tempo. Leach (1971), estudando o efeito da temperatura sobre o crescimento de brotos da alfafa, constatou um aumento de 38 para 50 brotos/planta quando a temperatura subiu de $15^{\circ} \mathrm{C}$ para $33^{\circ} \mathrm{C}$.

$\mathrm{O}$ desdobramento da interação entre cortes e doses de fósforo evidenciou um aumento no número de brotos da coroa $(\mathrm{P}<0,05)$ no terceiro corte para as doses de 100 e $200 \mathrm{mg} \mathrm{P} \mathrm{dm}^{-3}$ (Tabela 7). No entanto, isso não se repetiu com a dose de $50 \mathrm{mg} \mathrm{P} \mathrm{dm}^{-3}$, que foi responsável por um menor número $(\mathrm{P}<0,05)$ de brotos no terceiro corte (Tabela 7). Dessa maneira, o aumento do número de brotos no decorrer dos ciclos de crescimento da alfafa é dependente, também, do estado nutricional em que se encontra a planta. A redução na quantidade de brotos da planta devido à deficiência de nutrientes pode proporcionar menor longevidade da cultura. 
Tabela 7 - Número de brotos basais em alfafa obtidos nos três períodos de crescimento (cortes) em função das doses de fósforo (médias dos tratamentos com fontes de fósforo) e tratamentos com fontes de fósforo (médias de todas as doses)

Table 7 - Number of basal shoots in alfalfa obtained during three growth period (cuts) as a function of phosphorus rates (means by treatments with phosphorus sources) and with phosphorus sources treatments (means by phosphorus rates)

\begin{tabular}{|c|c|c|c|}
\hline \multirow[b]{2}{*}{$\begin{array}{l}\text { Doses de fósforo } \\
\text { Phosphorus rates }\end{array}$} & \multicolumn{3}{|c|}{$\begin{array}{l}\text { Cortes } \\
\text { Cuts }\end{array}$} \\
\hline & 1 & 2 & 3 \\
\hline $\begin{array}{l}\left(\mathrm{mg} \mathrm{dm}^{-3}\right) \\
\left(m g d m^{-3}\right)\end{array}$ & \multicolumn{3}{|c|}{$\begin{array}{l}\text { Número de brotos basais/planta } \\
\text { Number of basal shoot/plant }\end{array}$} \\
\hline $\begin{array}{l}50 \\
100 \\
200\end{array}$ & $\begin{array}{c}1,38^{\mathrm{ab}} \\
4,18^{\mathrm{c}} \\
7,88^{\mathrm{b}}\end{array}$ & $\begin{array}{l}1,65^{\mathrm{a}} \\
4,81^{\mathrm{b}} \\
6,87^{\mathrm{c}}\end{array}$ & $\begin{array}{l}1,48^{\mathrm{b}} \\
6,63^{\mathrm{a}} \\
9,23^{\mathrm{a}}\end{array}$ \\
\hline \multicolumn{4}{|c|}{$\begin{array}{l}\text { Tratamentos com fontes de fósforo } \\
\text { Treatments with phosphorus sources }\end{array}$} \\
\hline $\begin{array}{l}\text { STAC } \\
T S B L\end{array}$ & $3,47^{\mathrm{a}}$ & $3,90^{\mathrm{a}}$ & $4,13^{\mathrm{a}}$ \\
\hline $\begin{array}{l}\text { STDC } \\
\text { TSAL }\end{array}$ & $4,27^{\mathrm{a}}$ & $3,70^{\mathrm{a}}$ & $4,83^{\mathrm{a}}$ \\
\hline $\begin{array}{l}\text { FGAC } \\
G P B C\end{array}$ & $3,83^{\mathrm{a}}$ & $3,97^{\mathrm{a}}$ & $5,03^{\mathrm{a}}$ \\
\hline $\begin{array}{l}\text { FGDC } \\
G P A L\end{array}$ & $4,13^{b}$ & $4,47^{b}$ & $6,33^{\mathrm{a}}$ \\
\hline $\begin{array}{l}\text { FGACG } \\
G P B L C\end{array}$ & $5,47^{\mathrm{b}}$ & $5,23^{\mathrm{b}}$ & $7,23^{\mathrm{a}}$ \\
\hline $\begin{array}{l}\text { FGDCG } \\
G P A L C\end{array}$ & $5,73^{b}$ & $5,40^{\mathrm{b}}$ & $7,13^{\mathrm{a}}$ \\
\hline
\end{tabular}

STAC e STDC: super triplo antes e depois da calagem; FGAC e FGDC: fosfato de Gafsa antes e depois da calagem; FGACG e FGDCG: fosfato de Gafsa antes e depois da calagem mais gesso.

* Médias seguidas por letras distintas na mesma linha diferem entre si a $5 \%$ de probabilidade pelo teste de Tukey. CV (parcelas e subparcelas) $=11,4$ e $9,0 \%$, respectivamente.

* Médias seguidas por letras distintas na mesma linha diferem entre si a $5 \%$ de probabilidade pelo teste de Tukey. CV (parcelas e subparcelas) $=11,4$ e $9,0 \%$, respectivamente.

TSBL, TSAL: Triple superphosphate before and after liming; GPBL, GPAL: Gafsa phosphate before and after liming; GPBLG, GPALG: Gafsa phosphate before and afer liming combined with gypsum.

* Means followed by different letters within lines differ among them by Tukey test $(P<.05)$. CV (plots and subplots) $=11.4$ and $9.0 \%$, respectively.

${ }^{*}$ Means followed by different letters within lines differ among them by Tukey test $(P<.05)$. CV (plots and subplots) $=11.4$ and $9.0 \%$, respectively.

\section{Conclusões}

A utilização de adubação fosfatada em alfafa tem importante influência na velocidade de rebrota, assim como na quantidade de brotos produzidos pela planta. $\mathrm{O}$ fosfato de Gafsa foi uma fonte eficiente de fósforo quanto ao número de brotos de alfafa produzidos após cinco meses da mistura do adubo ao solo. O uso de gesso combinado ao fosfato de Gafsa foi benéfico para o aumento do número de brotos basais dessa leguminosa, enquanto que o momento de calagem não interferiu na brotação da planta.

\section{Literatura Citada}

BELESKY, D.P.; FEDDERS, J.M. Residue height influences stand dynamics of alfalfa grown on a Shallow soil. Agronomy Journal, v.89, n.6, p.975-980, 1997.
BRAGA, N.R.; MASCARENHAS, H.A.A.; BULISANI, E.A. et al. Eficiência agrônomica de nove fosfatos em quatro cultivos consecutivos de soja. Revista Brasileira de Ciência do Solo, v.15, p.315-319, 1991.

CANTARUTTI, R.B.; BRAGA, J.M.; NOVAIS, R.F. et al. Época de aplicação de fosfato natural em relação à calagem, num solo com elevado teor de alumínio trocável. Revista Brasileira de Ciência do Solo, v.5, p.129-133, 1981.

COWETT, E.R.; SPRAGUE, M.A. Factors affecting tillering in alfalfa. Agronomy Journal, v.54, n.4, p.294-297, 1962.

DA SILVA, S.C.; PEDREIRA, C.G.S. Princípios de ecologia aplicados ao manejo da pastagem. In: SIMPÓSIO SOBRE ECOSSISTEMA DE PASTAGENS, 3., 1997, Jaboticabal. Anais... Jaboticabal: Fundação Universidade Estadual Paulista, 1997. p.1-62.

DELISTOIANOV, J.; MATTOS, H.B.; MONTEIRO, F.A. Aplicação de fontes de fósforo e gesso em uma pastagem consorciada estabelecida em um Latossolo Vermelho-Escuro. Botetim da Indústria Animal, v.49, n.2, p.83-90, 1992.

FANQUIN, V.; PASSOS, R.R.; VILLA, M.R. et al. Absorção e acumulação de nutrientes por gramíneas forrageiras sob influência de fontes de fósforo e correção do solo. Revista Brasileira de Zootecnia, v.26, n.2, p.219-226, 1997. 
FICK, G.W.; HOLT, D.A.; LUGG, D.G. Environmental physiology and crop growth. In: HANSON, A.A.; BARNES, D.K.; HILL, R.R. (Eds.) Alfalfa and alfalfa improvement. Madison: ASA, CSSA, SSSA, 1988. p.163-194.

GOSSE, G.; LEMAIRE, G.; CHARTIER, M. Structure of a lucerne population (Medicago sativa L.) and dynamics of stem competition for light during regrowth. Journal of Applied Ecology, v.25, n.2, p.609-617, 1988.

LEACH, G.J. Shoot growth on lucerne plants cut at different heights. Australian Journal of Agricultural Research, v.21, n.4, p.583-691, 1970.

LEACH, G.J. The relation between lucerne shoot growth and temperature. Australian Journal of Agricultural Research, v.22, n.1, p.49-59, 1971.

MONTEIRO, A.L.G. Estudos morfológicos e fisiológicos da rebrota de cultivares não dormentes de alfafa (CUF 101 e Crioula) a partir do manejo de área foliar de perfilhos basilares. Piracicaba: Escola Superior de Agricultura "Luiz de Queiroz", 1989. 139p. Dissertação (Mestrado em Ciência Animal e Pastagens) - Escola Superior de Agricultura "Luiz de Queiroz", 1989.

MONTEIRO, A.L.G.; MORAES, A. Fisiologia e morfologia de plantas forrageiras. In: MONTEIRO, A.L.G.; MORAES, A.; CORRÊA, E.A.S. et al. (Eds.) Forragicultura no Paraná. Londrina: CPAF, 1996. p.75-92.

Van RAIJ, B.; ROSAND, P.C.; LOBATO, E. Adubação fosfatada no Brasil - apreciação geral, conclusões e recomendações. In: OLIVEIRA, A.J.; LOURENÇO, S.; GOEDERT, W.J. (Eds.) Adubação fosfatada no Brasil. Planaltina: EMBRAPA, CPAC, 1982. p.9-28. (Documentos, 21).
RANDO, E.M. Desenvolvimento da alfafa (Medicago sativa L.) em diferentes níveis de pH, potássio e enxofre no solo. Piracicaba: Escola de Agricultura "Luiz de Queiroz", 1992. 220p. Tese (Doutorado em Solos e Nutrição de Plantas) Escola de Agricultura "Luiz de Queiroz”, 1992.

ROMERO, N.A.; COMERÓN, E.A.; USTARROZ, E. Crecimiento y utilización de la alfalfa. In: HIJANO, E.H.; NAVARRO, A. (Eds.) La alfalfa en la Argentina. San Juan: Editar, 1995. p.149-172.

SANDERSON, M.A.; JONES, R.M. Stand dynamics and yield components of alfalfa as affected by phosphorus fertility. Agronomy Journal, v.85, n.2, p.241-246, 1993.

SARRIÉS, G.A.;ALVES, M.C.;OLIVEIRA,J.C.V.Sanest. Piracicaba: USP, ESALQ, CIAGRI, 1993. 57p. (Série Didática, 6).

TEUBER, L.R.; BRICK, M.A. Morphology and anatomy. In: HANSON, A.A.; BARNES, D.K.; HILL, R.R. (Eds.) Alfalfa and alfalfa improvement. Madison: ASA, CSSA, SSSA, 1988. p.125-162.

Recebido em: 28/08/01

Aceito em: 10/01/02 